\title{
Polar-Analytic Functions: Old and New Results, Applications
}

\author{
Carlo Bardaro, Paul L. Butzer, Ilaria Mantellini, and \\ Gerhard Schmeisser
}

Dedicated with great respect to the memory of Professor Heinrich Wefelscheid

\begin{abstract}
Here we review the notion of polar analyticity introduced in a previous paper and successfully applied in Mellin analysis and for quadrature formulae over the positive real axis. This approach provides a simple way of describing functions which are analytic on a part of the Riemann surface of the logarithm. New results are also obtained.
\end{abstract}

Mathematics Subject Classification. 30B10, 30E20, 30C20.

Keywords. Polar-analytic functions, Cauchy's integral formula, Morera's theorem, Taylor's expansion.

\section{Introduction and Motivation}

One of the main results of Fourier analysis is the Paley-Wiener theorem (see e.g. [17]). It characterizes the so-called bandlimited functions by entire functions of exponential type. More precisely, it states that a function $f \in L^{2}(\mathbb{R})$ has a Fourier transform $\hat{f}$ that vanishes outside a compact interval $[-\sigma, \sigma]$, where $\sigma>0$, if and only if $f$ has an extension to the whole complex plane as an entire function of exponential type $\sigma$, that is,

$$
|f(z)| \leq C \exp (\sigma|\Im z|) \quad(z \in \mathbb{C})
$$

with a constant $C>0$.

Carlo Bardaro and Ilaria Mantellini have been partially supported by the "Gruppo Nazionale per l'Analisi Matematica e Applicazioni (GNAMPA)" of the "Instituto di Alta Matematica (INDAM)" as well as by the project "Ricerca di Base 2019 of University of Perugia (title: Integrazione, Approssimazione, Analisi non lineare e loro Applicazioni)". 
This basic theorem has many applications, in particular in Shannon's sampling theory, the reproducing kernel theory, the investigation of quadrature formulae over the real line and various other topics.

During the nineties, P.L. Butzer and S. Jansche started a systematic study of the Mellin transform with the aim to establish a Mellin analysis as a fully independent counterpart of Fourier analysis (see $[10,11,13]$ ). Later on, many results were obtained in the framework of Mellin theory, especially contributions to approximation theory. One of the main motivations for this development relies on the exponential sampling theory, introduced in a formal way by optical physicists and engineers, due to its applications in light scattering, Fraunhofer diffraction, radio-astronomy and so on (see, e.g., [9]). Using Mellin analysis, Butzer and Jansche gave a rigorous version of the exponential sampling formula for functions defined over the positive real axis (see [12]). Later on, interconnections with other basic formulae were studied (see [1]). The exponential sampling formula in its classic form can be obtained under the assumption that the involved function $f$ is bandlimited in the Mellin transform sense. Therefore it was quite natural to investigate the structure of the space containing the Mellin bandlimited functions. This was done in several papers (see, e.g., [25]), but using a "real" approach involving certain differential operators, called transmutation operators. A fully complex version was given by Z. Szmydt and her school in Cracow (see, e.g., [23,24]) in which the authors characterize the Mellin transform of functions (distributions) with compact support. But, unlike what happens in Fourier analysis, the inverse of a Mellin transform is not a Mellin transform (in $L^{2}$ setting). Therefore the study of the structure of Mellin bandlimited functions requires a quite different approach.

A first complex result in this direction was obtained in [2] involving functions defined over the Riemann surface of the complex logarithm $S_{\log }$ and their analytic branches on it. Indeed, it was observed that a non-trivial, Mellin bandlimited function cannot be extended to the whole complex plane as an entire function, but it may be extended to $S_{\log }$ in such a way that the analytic branches $f_{k}$ are of exponential type in the sense that

$$
\left|f_{k}\left(r e^{i \theta}\right)\right| \leq C e^{\sigma|2 \pi k+\theta|} \quad(k \in \mathbb{Z}, \theta \in[0,2 \pi], r>0) .
$$

However, the handling of analytic branches is quite inconvenient for computational purposes. Therefore, in [3], we introduced a different approach, based on a simple geometric representation of the Riemann surface of the logarithm as a helicoidal surface in $\mathbb{R}^{3}$. Following this interpretation, we finally arrived at a new definition of analyticity, called polar analyticity, which uses the polar plane

$$
\mathbb{H}:=\{(r, \theta): r>0, \theta \in \mathbb{R}\}
$$


as a representation of $S_{\log }$. Here we note that this kind of representation of the Riemann surface was also considered, in different studies, in [19]. Further developments were presented in [6].

Our notion of polar analyticity is motivated by considering the complex variable $z$ in polar coordinates $z=r e^{i \theta}$ and treating $(r, \theta)$ as Cartesian coordinates. This modified notion of analyticity gives a simple description of analytic function on parts of the Riemann surface of the logarithm without employing branches. It also reveals a direct and simple applicability to several topics in Mellin analysis, from exponential sampling theory to quadrature formulae over the positive real axis (in this respect see the papers $[5,7,8]$ ). Moreover, it is useful in order to define function spaces like Hardy-type spaces in Mellin setting.

The present article gives a survey on the development of the theory of polar-analytic functions, including also some new results, obtaining counterparts of the Cauchy integral theorems, series expansions, conformality, residuetype theorems, identity principles, and so on. The new results involve polaranalytic versions of the maximum modulus principle, Liouville's theorem with an application to a version of the fundamental theorem of algebra for the socalled polar polynomials, and Rouché's theorem. The final sections are devoted to various applications in the framework of Mellin analysis. In particular, we describe applications to exponential sampling and to quadrature formulae. These applications testify the usefulness of this new theory.

\section{Basic Notions of Mellin Analysis}

In what follows, we denote by $\mathbb{N}, \mathbb{N}_{0}, \mathbb{Z}$, the sets of positive integers, nonnegative integers and integers, respectively, by $\mathbb{R}$ and $\mathbb{R}^{+}$, the sets of real and positive real numbers, respectively, and by $\mathbb{C}$ the set of complex numbers.

Let $C\left(\mathbb{R}^{+}\right)$be the space of all continuous functions defined on $\mathbb{R}^{+}$, and $C^{(r)}\left(\mathbb{R}^{+}\right)$be the space of all functions in $C\left(\mathbb{R}^{+}\right)$having a derivative of order $r$ that belongs to $C\left(\mathbb{R}^{+}\right)$. Analogously, by $C^{\infty}\left(\mathbb{R}^{+}\right)$we denote the space of all infinitely differentiable functions. By $L_{\text {loc }}^{1}\left(\mathbb{R}^{+}\right)$, we denote the space of all measurable functions which are integrable on every bounded interval in $\mathbb{R}^{+}$.

For $1 \leq p<+\infty$, let $L^{p}\left(\mathbb{R}^{+}\right)$be the space of all Lebesgue measurable and $p$-integrable complex-valued functions defined on $\mathbb{R}^{+}$endowed with the usual norm $\|f\|_{p}$. Analogous notations hold for functions defined on $\mathbb{R}$.

For $p=1$ and $c \in \mathbb{R}$, we introduce the space (see [10])

$$
X_{c}=\left\{f: \mathbb{R}^{+} \rightarrow \mathbb{C}: f(\cdot)(\cdot)^{c-1} \in L^{1}\left(\mathbb{R}^{+}\right)\right\}
$$

endowed with the norm

$$
\|f\|_{X_{c}}:=\left\|f(\cdot)(\cdot)^{c-1}\right\|_{1}=\int_{0}^{+\infty}|f(u)| u^{c-1} d u .
$$


More generally, let $X_{c}^{p}$ denote the space of all functions $f: \mathbb{R}^{+} \rightarrow \mathbb{C}$ such that $f(\cdot)(\cdot)^{c-1 / p} \in L^{p}\left(\mathbb{R}^{+}\right)$with $1<p<\infty$. Finally, for $p=\infty$, we define $X_{c}^{\infty}$ as the space comprising all measurable functions $f: \mathbb{R}^{+} \rightarrow \mathbb{C}$ such that $\|f\|_{X_{c}^{\infty}}:=\sup _{x>0} x^{c}|f(x)|<\infty$.

The Mellin translation operator $\tau_{h}^{c}$ for $h \in \mathbb{R}^{+}, c \in \mathbb{R}$ and $f: \mathbb{R}^{+} \rightarrow \mathbb{C}$ is defined by

$$
\left(\tau_{h}^{c} f\right)(x):=h^{c} f(h x) \quad\left(x \in \mathbb{R}^{+}\right) .
$$

Setting $\tau_{h}:=\tau_{h}^{0}$, we have $\left(\tau_{h}^{c} f\right)(x)=h^{c}\left(\tau_{h} f\right)(x)$ and $\left\|\tau_{h}^{c} f\right\|_{X_{c}}=\|f\|_{X_{c}}$.

In the Mellin frame, the natural concept of a pointwise derivative of a function $f$ is given by the limit of the difference quotient involving the Mellin translation; thus if $f^{\prime}$ exists, then

$$
\lim _{h \rightarrow 1} \frac{\tau_{h}^{c} f(x)-f(x)}{h-1}=x f^{\prime}(x)+c f(x) .
$$

This gives the motivation for the following definition (see [10]): The pointwise Mellin differential operator $\Theta_{c}$ and the pointwise Mellin derivative $\Theta_{c} f$ of a function $f: \mathbb{R}^{+} \rightarrow \mathbb{C}$ and $c \in \mathbb{R}$ are defined by

$$
\Theta_{c} f(x):=x f^{\prime}(x)+c f(x) \quad\left(x \in \mathbb{R}^{+}\right)
$$

provided that $f^{\prime}$ exists a.e. on $\mathbb{R}^{+}$. The Mellin differential operator of order $r \in \mathbb{N}$ is defined recursively by

$$
\Theta_{c}^{1}:=\Theta_{c}, \quad \Theta_{c}^{r}:=\Theta_{c}\left(\Theta_{c}^{r-1}\right) .
$$

For convenience, we set $\Theta^{r}:=\Theta_{0}^{r}$ and $\Theta_{c}^{0}:=I$ with $I$ denoting the identity operator.

The Mellin transform of a function $f \in X_{c}$ is the linear and bounded operator defined by (see, e.g., [10])

$$
M_{c}[f](s) \equiv[f]_{M_{c}}^{\wedge_{c}}(s):=\int_{0}^{+\infty} u^{s-1} f(u) d u \quad(s=c+i t, t \in \mathbb{R}) .
$$

The inverse Mellin transform $M_{c}^{-1}[g]$ of a function $g \in L^{1}(c+i \mathbb{R})$ is defined by

$$
M_{c}^{-1}[g](x):=\frac{x^{-c}}{2 \pi} \int_{-\infty}^{+\infty} g(c+i t) x^{-i t} d t \quad\left(x \in \mathbb{R}^{+}\right),
$$

where in general $L^{p}(c+i \mathbb{R})$, for $p \geq 1$, will mean the space of all functions $g: c+i \mathbb{R} \rightarrow \mathbb{C}$ with $g(c+i \cdot) \in L^{p}(\mathbb{R})$.

More generally, for $1<p \leq 2$, the Mellin transform $M_{c}^{p}$ of $f \in X_{c}^{p}$ is given by (see [13] for $p=2$ )

$$
M_{c}^{p}[f](s) \equiv[f]_{M_{c}^{p}}^{\wedge}(s)=\text { l.i.m. } \rho \rightarrow+\infty \int_{1 / \rho}^{\rho} f(u) u^{s-1} d u,
$$


for $s=c+i t$, in the sense that

$$
\lim _{\rho \rightarrow \infty}\left\|M_{c}^{p}[f](c+i t)-\int_{1 / \rho}^{\rho} f(u) u^{c+i t-1} d u\right\|_{L^{p^{\prime}}(c+i \mathbb{R})}=0,
$$

where $p^{\prime}$ is the conjugate exponent of $p$, that is, $1 / p+1 / p^{\prime}=1$.

Analogously, for example for $p=2$, we define the inverse Mellin transform of a function $g \in L^{2}(c+i \mathbb{R})$ by

$$
M_{c}^{2,-1}[g](x)=\text { l.i.m. } \rho \rightarrow+\infty \frac{1}{2 \pi} \int_{-\rho}^{\rho} g(c+i t) x^{-c-i t} d t,
$$

where the limit is taken in $X_{c}^{2}$-norm. For any $f \in X_{c}^{2}$, there holds

$$
M_{c}^{2,-1}\left[M_{c}^{2}[f]\right](x)=f(x) \quad\left(\text { a.e. on } \mathbb{R}^{+}\right),
$$

see $[13]$.

\section{Polar-Analytic Functions}

In this section, we will describe a new concept of analyticity which turns out to be very useful in the development of Mellin analysis. We begin with some preliminary facts concerning complex functions in polar coordinates.

Let us first recall that in classical complex analysis the Cauchy-Riemann equations in polar coordinates are obtained by setting $z=r e^{i \theta}$, i.e., given an analytic function $f=u+i v$ on a domain in the complex plane, one considers the function $g(r, \theta):=f\left(r e^{i \theta}\right)$ and uses the chain rule for deriving partial derivatives of $u, v$ with respect to the variables $r, \theta$ (see, e.g., [18]). Due to the $2 \pi$-periodicity of the exponential function $e^{i \theta}$, this implies a periodicity with respect to $\theta$ of the function $g$. As we shall see, in our definition of polar analyticity, we again derive the same Cauchy-Riemann equations in polar form, but in general this periodicity does not appear. As a consequence, this approach gives a simple description of analytic functions defined on a part of the Riemann surface of the complex logarithm without involving branches.

\subsection{Definition and Elementary Properties}

Let $\mathbb{H}:=\left\{(r, \theta) \in \mathbb{R}^{+} \times \mathbb{R}\right\}$ be the right half-plane. By a domain in $\mathbb{H}$, we mean a non-empty, open and connected subset of $\mathbb{H}$. Let $\mathscr{D}$ be a domain in $\mathbb{H}$.

Definition 1. We say that $f: \mathscr{D} \rightarrow \mathbb{C}$ is polar-analytic on $\mathscr{D}$ if for any $\left(r_{0}, \theta_{0}\right) \in$ $\mathscr{D}$ the limit

$$
\lim _{(r, \theta) \rightarrow\left(r_{0}, \theta_{0}\right)} \frac{f(r, \theta)-f\left(r_{0}, \theta_{0}\right)}{r e^{i \theta}-r_{0} e^{i \theta_{0}}}=:\left(D_{\mathrm{pol}} f\right)\left(r_{0}, \theta_{0}\right)
$$

exists and is the same howsoever $(r, \theta)$ approaches $\left(r_{0}, \theta_{0}\right)$ within $\mathscr{D}$. 
Polar analyticity of two functions is inherited by their arithmetic combinations and the familiar rules known for classical differentiation hold for polar derivatives as well. For details see [6].

Let $f:(r, \theta) \mapsto u(r, \theta)+i v(r, \theta)$ be polar-analytic on $\mathscr{D}$, where $u$ and $v$ are real-valued. Identifying $\mathbb{C}$ with $\mathbb{R}^{2}$, we may interpret $f$ as a mapping from a subset of the half-plane $\mathbb{H}$ into $\mathbb{R}^{2}$. Then we have (see [6]):

Proposition 1. A polar-analytic function is differentiable in the classical sense.

It can be verified that $f=u+i v$ with $u, v: \mathscr{D} \rightarrow \mathbb{R}$ is polar-analytic on $\mathscr{D}$ if and only if $u$ and $v$ have continuous partial derivatives on $\mathscr{D}$ that satisfy the differential equations

$$
\frac{\partial u}{\partial \theta}=-r \frac{\partial v}{\partial r}, \quad \frac{\partial v}{\partial \theta}=r \frac{\partial u}{\partial r} .
$$

Note that these equations coincide with the Cauchy-Riemann equations of an analytic function $g$ defined by $g(z):=u(r, \theta)+i v(r, \theta)$ for $z=r e^{i \theta}$. For the derivative $D_{\text {pol }}$, we easily find that

$$
\begin{aligned}
\left(D_{\mathrm{pol}} f\right)(r, \theta) & =e^{-i \theta}\left(\frac{\partial}{\partial r} u(r, \theta)+i \frac{\partial}{\partial r} v(r, \theta)\right) \\
& =\frac{e^{-i \theta}}{r}\left(\frac{\partial}{\partial \theta} v(r, \theta)-i \frac{\partial}{\partial \theta} u(r, \theta)\right) .
\end{aligned}
$$

Since $f=u+i v$, equations (1) can be written in a more compact way as

$$
\frac{\partial f}{\partial \theta}=i r \frac{\partial f}{\partial r}
$$

and then formula (2) takes the form

$$
\left(D_{\text {pol }} f\right)(r, \theta)=e^{-i \theta} \frac{\partial}{\partial r} f(r, \theta)=\frac{e^{-i \theta}}{i r} \frac{\partial}{\partial \theta} f(r, \theta) .
$$

Also note that $D_{\text {pol }}$ is the ordinary differentiation on $\mathbb{R}^{+}$. More precisely, if $\varphi(\cdot):=f(\cdot, 0)$, then $\left(D_{\mathrm{pol}} f\right)(r, 0)=\varphi^{\prime}(r)$.

When $g$ is an entire function, then $f:(r, \theta) \mapsto g\left(r e^{i \theta}\right)$ defines a function $f$ on $\mathbb{H}$ that is polar-analytic and $2 \pi$-periodic with respect to $\theta$. Moreover, by (2) one has $\left(D_{\text {pol }} f\right)(r, \theta)=g^{\prime}(z)$ with $z=r e^{i \theta}$. A converse statement is not true in general. If $f$ is polar-analytic on $\mathbb{H}$ and $2 \pi$-periodic with respect to the second variable, there may not exist an entire function $h$ such that $f(r, \theta)=h\left(r e^{i \theta}\right)$. A simple example is the function $f(r, \theta):=e^{-i \theta} / r$. It would imply that $h(z)=1 / z$, which is analytic on $\mathbb{C} \backslash\{0\}$ only. However, if $f$ is a polar-analytic function on $\mathbb{H}$, then $g: z=x+i y \mapsto f\left(e^{x}, y\right)$ is an entire function.

As examples, let us consider the function $g(z)=z^{a}, a>0$. Defining $f(r, \theta):=g\left(r e^{i \theta}\right)=r^{a} e^{i a \theta}$, we have

$$
f(r, \theta)=u(r, \theta)+i v(r, \theta):=r^{a} \cos (a \theta)+i r^{a} \sin (a \theta)
$$


and so

$$
\left(D_{\text {pol }} f\right)(r, \theta)=e^{-i \theta}\left[\frac{\partial}{\partial r} u(r, \theta)+i \frac{\partial}{\partial r} v(r, \theta)\right]=a\left(r e^{i \theta}\right)^{a-1}=g^{\prime}(z) .
$$

Analogously, taking $g(z)=\sin z$ and $f(r, \theta):=\sin \left(r e^{i \theta}\right)$, we have $\left(D_{\text {pol }} f\right)(r, \theta)$ $=\cos \left(r e^{i \theta}\right)=g^{\prime}(z)$.

The main novelty of the definition of polar-analytic function is that, using this approach, we avoid periodicity with respect to the argument $\theta$, and in this way we can avoid the use of Riemann surfaces.

A simple example of a polar-analytic function that is not $2 \pi$-periodic is the function $L(r, \theta):=\log r+i \theta$, which is easily seen to satisfy the differential equations (1). In this approach, we consider the logarithm as a single-valued function on $\mathbb{H}$, without the use of the Riemann surface $S_{\log }$. Employing (2) and setting $z=r e^{i \theta}$, we find that

$$
\left(D_{\mathrm{pol}} L\right)(r, \theta)=e^{-i \theta} \frac{1}{r}=\frac{1}{r e^{i \theta}}=\frac{1}{z} .
$$

In order to state a connection with analytic functions on $S_{\log }$, for $\alpha, \beta \in \mathbb{R}$ with $\alpha<\beta$, we consider the set

$$
\mathbb{H}_{\alpha, \beta}:=\left\{(r, \theta) \in \mathbb{R}^{+} \times \mathbb{R}: \theta \in\right] \alpha, \beta[\}
$$

and call it a strip of $\mathbb{H}$.

If $f: \mathbb{H} \rightarrow \mathbb{C}$ is polar-analytic but not $2 \pi$-periodic with respect to $\theta$, then we can associate with $f$ a function $g$ that is analytic on the Riemann surface $S_{\log }$ of the logarithm. The restriction of $f$ to a strip $\mathbb{H}_{\alpha+2 k \pi, \alpha+2(k+1) \pi}$, where $k \in \mathbb{Z}$, defines an analytic function $g_{k}$ in the slit complex plane $\mathbb{C} \backslash\left\{r e^{i \alpha}: r>0\right\}$ by setting $g_{k}\left(r e^{i \theta}\right):=f(r, \theta)$. The functions $g_{k}$, for $k \in \mathbb{Z}$, are the analytic branches of $g$.

As to the geometric properties of polar-analytic functions, we recall that a remarkable geometric property of an analytic function $g$ in the classical sense is that at any point $z_{0}$ with $g^{\prime}\left(z_{0}\right) \neq 0$ it preserves angles and orientation. More precisely, this means the following. Let $\gamma_{1}$ and $\gamma_{2}$ be smooth arcs that intersect at $z_{0}$ where they have tangents $\mathbf{t}_{1}$ and $\mathbf{t}_{2}$, respectively. Suppose that we have to rotate $\mathbf{t}_{1}$ in the mathematically positive sense by an angle $\alpha$ around $z_{0}$ in order that it coincides with $\mathbf{t}_{2}$. Then the same is true for the $\operatorname{arcs} g \circ \gamma_{1}$ and $g \circ \gamma_{2}$, that is, if $\tau_{1}$ and $\tau_{2}$, respectively, are their tangents in $g\left(z_{0}\right)$, then we have to rotate $\tau_{1}$ in the mathematically positive sense by the angle $\alpha$ around $g\left(z_{0}\right)$ in order that it coincides with $\tau_{2}$. We cannot expect that this property extends to polar-analytic functions.

The following propositions inform us about the different geometric behavior (for the details see [6]).

Proposition 2. Let $f$ be polar-analytic in a neighborhood of a point $\left(r_{0}, \theta_{0}\right) \in \mathbb{H}$, and suppose that $\left(D_{\mathrm{pol}} f\right)\left(r_{0}, \theta_{0}\right) \neq 0$. Let $\gamma_{1}$ and $\gamma_{2}$ be two smooth arcs that intersect at $\left(r_{0}, \theta_{0}\right)$ under an angle $\alpha$. Then the arcs $f \circ \gamma_{1}$ and $f \circ \gamma_{2}$ intersect 
at $f\left(r_{0}, \theta_{0}\right)$ under an angle $\beta$ that depends on the local geometric situation in $\mathbb{H}$ but does not depend on $f$ and $\theta_{0}$. Furthermore, $\beta=\alpha$ when $r_{0}=1$.

Proposition 3. Let $\mathscr{D}$ be a domain in $\mathbb{H}$, and let $f: \mathscr{D} \rightarrow \mathbb{C}$ be polar-analytic with $D_{\text {pol }} f \neq 0$. Denote by $\mathscr{N}$ an orthogonal net of lines parallel to the axes of $\mathbb{H}$. Then $f$ maps $\mathscr{N} \cap \mathscr{D}$ onto an orthogonal net of curves in $f(\mathscr{D})$.

Examples are discussed in [6].

According to the notion of Mellin derivatives, we give the following definition (see [6]).

Definition 2. For a fixed real number $c \in \mathbb{R}$, we define the Mellin polar derivative as

$$
\widetilde{\Theta}_{c} f(r, \theta):=r e^{i \theta}\left(D_{\mathrm{pol}}\right) f(r, \theta)+c f(r, \theta),
$$

provided that the polar derivative $D_{\text {pol }} f$ exists at the point $(r, \theta) \in \mathbb{H}$.

Also in this case, note that for $\varphi(r):=f(r, 0)$, we have $\widetilde{\Theta}_{c} f(r, 0)=$ $\left(\Theta_{c} \varphi\right)(r)$, where $\Theta_{c}$ is the usual Mellin differential operator.

\section{Analysis of Polar-Analytic Functions}

In this section, we give counterparts of the classical Cauchy integral theorems and formulae, and a Taylor-like expansion. Concerning the Cauchy integral theorems, the key result is given in the subsequent Theorem 1. It was proved in [3] for convex domains only since this sufficed for the envisaged applications. However, that approach extends to simply connected domains as may be seen by employing [16, Theorem 8.4, page 372].

\subsection{Cauchy and Morera Type Theorems}

The following theorem on line integrals for polar-analytic functions includes an analogue of Cauchy's fundamental theorem of complex function theory. Here a piecewise continuously differentiable curve will be called a regular curve.

Theorem 1. Let $f$ be a polar-analytic function on a simply connected domain $\mathscr{D} \subset \mathbb{H}$ and let $\left(r_{1}, \theta_{1}\right)$ and $\left(r_{2}, \theta_{2}\right)$ be any two points in $\mathscr{D}$. Then the line integral

$$
\int_{\gamma} f(r, \theta) e^{i \theta}(d r+i r d \theta)
$$

has the same value for each regular curve $\gamma$ in $\mathscr{D}$ that starts at $\left(r_{1}, \theta_{1}\right)$ and ends $\left(r_{2}, \theta_{2}\right)$. In particular, the integral vanishes for closed regular curves.

As a consequence, we obtain the following result (see [3, Lemma 5.1]). 
Theorem 2. Let $\mathscr{D}$ be a domain in $\mathbb{H}$. Let $g: \mathscr{D} \rightarrow \mathbb{C}$ be continuous on the whole of $\mathscr{D}$ and polar-analytic except at a point $\left(r_{0}, \theta_{0}\right) \in \mathscr{D}$. Suppose that $\gamma$ is a positively oriented, closed, regular curve in $\mathscr{D}$ that is the boundary of a simply connected domain int $(\gamma)$. Then

$$
\int_{\gamma} g(r, \theta) e^{i \theta}(d r+i r d \theta)=0 .
$$

We can also establish a converse of Theorem 1 in the spirit of Morera's theorem (see [3]). For sake of simplicity, we consider functions defined on the whole of $\mathbb{H}$.

Theorem 3. Let $f: \mathbb{H} \rightarrow \mathbb{C}$ be a continuous function. If the integral (4) is zero on the boundary of every rectangle $R \subset \mathbb{H}$ with sides parallel to the axes, then $f$ is polar-analytic on $\mathbb{H}$.

\subsection{Cauchy Integral Formulae for Polar-Analytic Functions}

In this section, we report on an analogue of the classical Cauchy integral formula. For details see [6].

Theorem 4. Let $\mathscr{D}$ be a domain in $\mathbb{H}$, and let $f: \mathscr{D} \rightarrow \mathbb{C}$ be polar-analytic on $\mathscr{D}$. Let $\gamma$ be a positively oriented, closed, regular curve that is the boundary of a simply connected domain $\operatorname{int}(\gamma) \subset \mathscr{D}$. Given $\left(r_{0}, \theta_{0}\right) \in \operatorname{int}(\gamma)$, define $\theta_{j}:=\theta_{0}+2 j \pi$ for $j \in \mathbb{Z}$. Suppose that none of the points $\left(r_{0}, \theta_{j}\right)$ lies on $\gamma$. Then

$$
\frac{1}{2 \pi i} \int_{\gamma} \frac{f(r, \theta) e^{i \theta}}{r e^{i \theta}-r_{0} e^{i \theta_{0}}}(d r+i r d \theta)=\sum_{\left(r_{0}, \theta_{j}\right) \in \operatorname{int}(\gamma)} f\left(r_{0}, \theta_{j}\right)
$$

In particular, if $\gamma$ lies in a strip $\mathbb{H}_{a, b}$ with $0<b-a<2 \pi$, then

$$
\frac{1}{2 \pi i} \int_{\gamma} \frac{f(r, \theta) e^{i \theta}}{r e^{i \theta}-r_{0} e^{i \theta_{0}}}(d r+i r d \theta)=f\left(r_{0}, \theta_{0}\right) .
$$

An interesting consequence of Theorem 4 is the following representation formula for polar derivatives:

Theorem 5. Under the hypotheses of Theorem 4, if $\gamma$ lies in a strip $\mathbb{H}_{a, b}$ with $0<b-a<2 \pi$, then

$$
\left(D_{\mathrm{pol}}^{k} f\right)(r, \theta)=\frac{k !}{2 \pi i} \int_{\gamma} \frac{f(\rho, \phi) e^{i \phi}}{\left(\rho e^{i \phi}-r e^{i \theta}\right)^{k+1}}(d \rho+i \rho d \phi) \quad((r, \theta) \in \operatorname{int}(\gamma), k \in \mathbb{N}) .
$$

\subsection{A Series Expansion for Polar-Analytic Functions}

In this section, we present a Taylor-like series expansions for a polar-analytic function. As we shall see, this expansion exists on strips of width less than $2 \pi$ only. It amounts to taking an analytic branch on the Riemann surface of the logarithm and expanding it in the classical way. 
In the next section, we will obtain another expansion involving the Mellin polar derivatives which has a global character, that is, it may exists on the whole of $\mathbb{H}$.

Given a curve $\gamma \operatorname{such}$ that $\operatorname{int}(\gamma)$ is simply connected and $\left(r_{0}, \theta_{0}\right) \in \operatorname{int}(\gamma)$, let us define

$$
\mathscr{D}\left(r_{0}, \theta_{0} ; \gamma\right):=\left\{(r, \theta) \in \operatorname{int}(\gamma):\left|r e^{i \theta}-r_{0} e^{i \theta_{0}}\right|<\min _{(\rho, \phi) \in \gamma}\left|\rho e^{i \phi}-r_{0} e^{i \theta_{0}}\right|\right\} .
$$

The following Taylor-like expansion of polar-analytic functions holds (see [6]).

Theorem 6. Under the assumptions of Theorem 5, we have

$$
f(r, \theta)=\sum_{k=0}^{\infty} \frac{D_{\text {pol }}^{k} f\left(r_{0}, \theta_{0}\right)}{k !}\left(r e^{i \theta}-r_{0} e^{i \theta_{0}}\right)^{k}
$$

for $(r, \theta) \in \mathscr{D}\left(r_{0}, \theta_{0} ; \gamma\right)$.

\section{Polar-Analytic Functions in Mellin Setting}

In this section, we describe an analysis of polar-analytic functions in Mellin setting. A motivation comes from the fact that our analogue of Cauchy's integral formula of Theorem 4 is useful in horizontal strips of width less than $2 \pi$ only since otherwise it may produce additional residues as undesired artifacts. Therefore one of the aims of this section is to establish a further analogue of Cauchy's integral formula which is always free of artifacts, and a version of the residue theorem for polar-analytic functions.

\subsection{A Series Expansion with Mellin Polar Derivatives}

We premise some notations and remarks.

For $z_{0}=x_{0}+i y_{0} \in \mathbb{C}$ and $\rho>0$, consider the disk $D\left(z_{0}, \rho\right):=\{z \in$ $\left.\mathbb{C}:\left|z-z_{0}\right|<\rho\right\}$ in $\mathbb{C}$. By $x+i y \mapsto(\log r, \theta)$, where $r=e^{x}$ and $y=\theta$, it is transformed into the region with one axis of symmetry in $\mathbb{H}$, given by

$$
E\left(\left(r_{0}, \theta_{0}\right), \rho\right):=\left\{(r, \theta) \in \mathbb{H}:\left(\log \frac{r}{r_{0}}\right)^{2}+\left(\theta-\theta_{0}\right)^{2}<\rho^{2}\right\},
$$

which we call a polar-disk with center $\left(r_{0}, \theta_{0}\right)$ and radius $\rho$. Here $r_{0}=e^{x_{0}}$ and $\theta_{0}=y_{0}$. The boundary of this region is given by the graph of the functions

$$
r=r_{0} \exp \left( \pm \sqrt{\rho^{2}-\left(\theta-\theta_{0}\right)^{2}}\right), \quad \theta \in\left[-\rho+\theta_{0}, \rho+\theta_{0}\right] .
$$

Given a domain $\mathscr{D} \subset \mathbb{H}$, we define

$$
A:=\left\{z=x+i y \in \mathbb{C}:\left(e^{x}, y\right) \in \mathscr{D}\right\} .
$$


Now for $z_{0}=x_{0}+i y_{0} \in A$, consider a disk $D\left(z_{0}, \rho\right) \subset A$. Then, in order that the associated polar-disk $E\left(\left(r_{0}, \theta_{0}\right), \rho\right)$ is fully contained in $\mathscr{D}$, the maximal admissible $\rho>0$ is given by

$$
\rho=\min _{(r, \theta) \in \partial \mathscr{D}} \sqrt{\left(\log \frac{r}{r_{0}}\right)^{2}+\left(\theta-\theta_{0}\right)^{2}},
$$

where $\partial \mathscr{D}$ denotes the boundary of $\mathscr{D}$.

The following theorem gives a "global" version of the series expansion of the previous section and uses Mellin polar derivatives (see $[6,7]$ ).

Theorem 7. Let $f: \mathscr{D} \rightarrow \mathbb{C}$ be polar-analytic on a domain $\mathscr{D} \subset \mathbb{H}$. If $\left(r_{0}, \theta_{0}\right) \in$ $\mathscr{D}$, then there holds the expansion

$$
f(r, \theta)=\sum_{k=0}^{\infty} \frac{\left(\widetilde{\Theta}_{0}^{k} f\right)\left(r_{0}, \theta_{0}\right)}{k !}\left(\log \frac{r}{r_{0}}+i\left(\theta-\theta_{0}\right)\right)^{k}
$$

converging on every polar-disk $E\left(\left(r_{0}, \theta_{0}\right), \rho\right) \subset \mathscr{D}$, the convergence being uniform on compact subsets of these polar-disks.

A slight extension of the above expansion is given by the following theorem (see [7]).

Theorem 8. Let $f: \mathscr{D} \rightarrow \mathbb{C}$ be polar-analytic on a domain $\mathscr{D} \subset \mathbb{H}$ and let $c \in \mathbb{R}$. If $\left(r_{0}, \theta_{0}\right) \in \mathscr{D}$, then there holds the expansion

$$
\left(r e^{i \theta}\right)^{c} f(r, \theta)=\left(r_{0} e^{i \theta_{0}}\right)^{c} \sum_{k=0}^{\infty} \frac{\left(\widetilde{\Theta}_{c}^{k} f\right)\left(r_{0}, \theta_{0}\right)}{k !}\left(\log \frac{r}{r_{0}}+i\left(\theta-\theta_{0}\right)\right)^{k}
$$

converging on every polar-disk $E\left(\left(r_{0}, \theta_{0}\right), \rho\right) \subset \mathscr{D}$, the convergence being uniform on compact subsets of these polar-disks.

A first remarkable consequence of Theorem 8 is the following identity theorem for polar-analytic functions (see [7]).

Theorem 9. Let $\mathscr{D}$ be a domain in $\mathbb{H}$ and let $f: \mathscr{D} \rightarrow \mathbb{C}$ be polar-analytic. Suppose that $\left(r_{0}, \theta_{0}\right) \in \mathscr{D}$ is an accumulation point of distinct zeros of $f$. Then $f$ is identically zero.

\subsection{A General Cauchy Integral Formula for Polar-Analytic Functions in Mellin Setting}

A second fundamental consequence of Theorem 8 is given by a general version of Cauchy's integral formula for Mellin polar derivatives (see [7]).

Theorem 10. Let $\mathscr{D}$ be a domain in $\mathbb{H}$, and let $f: \mathscr{D} \rightarrow \mathbb{C}$ be polar-analytic on $\mathscr{D}$. Let $\gamma$ be a positively oriented, closed, regular curve that is the boundary 
of a simply connected domain $\operatorname{int}(\gamma) \subset \mathscr{D}$. Then, for $\left(r_{0}, \theta_{0}\right) \in \operatorname{int}(\gamma), c \in \mathbb{R}$ and $k \in \mathbb{N}_{0}$, we have

$$
\frac{1}{2 \pi i} \int_{\gamma} \frac{\left(r e^{i \theta}\right)^{c-1} f(r, \theta) e^{i \theta}}{\left(\log \left(r / r_{0}\right)+i\left(\theta-\theta_{0}\right)\right)^{k+1}}(d r+i r d \theta)=\left(r_{0} e^{i \theta_{0}}\right)^{c} \frac{\left(\widetilde{\Theta}_{c}^{k} f\right)\left(r_{0}, \theta_{0}\right)}{k !}
$$

Other than the result of Theorem 5, the previous formula is also useful without a restriction to horizontal strips of width less than $2 \pi$ since it does not produce additional residues as happens before.

\subsection{A Residue Theorem for Polar-Analytic Functions in Mellin Setting}

Here we introduce the notion of pole in its logarithmic representation and give a version of the residue theorem for polar-analytic functions which have poles as singularities; see [7].

Definition 3. Let $\left(r_{0}, \theta_{0}\right) \in \mathbb{H}$ and let $\mathscr{U} \subset \mathbb{H}$ be an open neighborhood of $\left(r_{0}, \theta_{0}\right)$.

(i) If $f: \mathscr{U} \backslash\left\{\left(r_{0}, \theta_{0}\right)\right\} \rightarrow \mathbb{C}$ is polar-analytic, then $\left(r_{0}, \theta_{0}\right)$ will be called an isolated singularity of $f$.

(ii) An isolated singularity $\left(r_{0}, \theta_{0}\right)$ is said to be a pole of order $k$ if $k \in \mathbb{N}$ and there exists a polar-analytic function $g: \mathscr{U} \rightarrow \mathbb{C}$ with $g\left(r_{0}, \theta_{0}\right) \neq 0$ such that

$$
f(r, \theta)=\frac{g(r, \theta)}{\left(\log \left(r / r_{0}\right)+i\left(\theta-\theta_{0}\right)\right)^{k}} \quad \text { for }(r, \theta) \in \mathscr{U} \backslash\left\{\left(r_{0}, \theta_{0}\right)\right\} .
$$

In this case

$$
\left(\operatorname{res}_{c} f\right)\left(r_{0}, \theta_{0}\right):=\left(r_{0} e^{i \theta_{0}}\right)^{c} \frac{\left(\widetilde{\Theta}_{c}^{k-1} g\right)\left(r_{0}, \theta_{0}\right)}{(k-1) !}
$$

will be called the $c$-residue of $f$ at $\left(r_{0}, \theta_{0}\right)$.

We are now in a position to formulate a residue theorem for poles which will be suitable for many applications in Mellin analysis.

Theorem 11. Let $\mathscr{D}$ be a domain in $\mathbb{H}$ and let $f$ be polar-analytic on $\mathscr{D}$ except for isolated singularities which are all poles. Let $\gamma$ be a positively oriented, closed, regular curve that is the boundary of a simply connected domain $\operatorname{int}(\gamma) \subset \mathscr{D}$. Suppose that no isolated singularity lies on $\gamma$ while $\left(r_{j}, \theta_{j}\right)$ for $j=1, \ldots, m$ are the isolated singularities lying in $\operatorname{int}(\gamma)$. Then, for $c \in \mathbb{R}$, there holds

$$
\int_{\gamma}\left(r e^{i \theta}\right)^{c-1} f(r, \theta) e^{i \theta}(d r+i r d \theta)=2 \pi i \sum_{j=1}^{m}\left(\operatorname{res}_{c} f\right)\left(r_{j}, \theta_{j}\right) .
$$




\subsection{Further Developments}

Here we obtain some new results about polar-analytic functions in Mellin setting. In particular, we discuss polar Mellin versions of the classical maximum modulus principle, of Liouville's theorem and of the fundamental theorem of algebra for polar polynomials. Note that our proofs are independent of the classical complex analysis and use only tools from the theory of polar-analytic functions developed previously.

Theorem 12. Under the assumptions of Theorem 10, we have

$$
|f(r, \theta)| \leq \max _{(\rho, \vartheta) \in \gamma}|f(\rho, \vartheta)| \quad \text { for }(r, \theta) \in \operatorname{int}(\gamma)
$$

Proof. Writing $M:=\max _{(\rho, \vartheta) \in \gamma}|f(\rho, \vartheta)|$, we assume for a contradiction that there exists a point $\left(r_{0}, \theta_{0}\right) \in \operatorname{int}(\gamma)$ such that $\left|f\left(r_{0}, \theta_{0}\right)\right|>M$. Since for any $n \in \mathbb{N}$ the function $f^{n}$ also satisfies the assumptions of Theorem 10, we have by (10) with $k=c=0$ that

$$
\left(f\left(r_{0}, \theta_{0}\right)\right)^{n}=\frac{1}{2 \pi i} \int_{\gamma} \frac{(f(r, \theta))^{n}}{r\left(\log \left(r / r_{0}\right)+i\left(\theta-\theta_{0}\right)\right)}(d r+i r d \theta) .
$$

Let $\gamma(t):=(\rho(t), \vartheta(t)), t \in[0,1]$, be a representation of $\gamma$, where $\rho(\cdot)$ and $\vartheta(\cdot)$ are piecewise continuously differentiable functions. Then, by (11), we can write

$$
\left|f\left(r_{0}, \theta_{0}\right)\right|^{n} \leq \frac{M^{n}}{2 \pi} \int_{0}^{1} \frac{\left|\rho^{\prime}(t)+i \rho(t) \vartheta^{\prime}(t)\right|}{\rho(t)\left|\log \left(\rho(t) / r_{0}\right)+i\left(\vartheta(t)-\theta_{0}\right)\right|} d t .
$$

The integral on the right-hand side does not depend on $n$. Hence taking the $n$-th root on both sides and letting $n \rightarrow \infty$, we obtain $\left|f\left(r_{0}, \theta_{0}\right)\right| \leq M$; a contradiction.

Now, using Theorem 12, we can derive an analogue of Liouville's theorem for polar-analytic functions. We shall say that $f$ is polar-entire if it is polaranalytic on the whole of $\mathbb{H}$.

Theorem 13. Let $f: \mathbb{H} \rightarrow \mathbb{C}$ be a bounded and polar-entire function. Then $f$ is constant.

Proof. Let $M>0$ be such that $|f(r, \theta)| \leq M$ for every $(r, \theta) \in \mathbb{H}$. By Theorem 8 , for $c=0$, we have the expansion

$$
f(r, \theta)=\sum_{k=0}^{\infty} \frac{\widetilde{\Theta}^{k} f(1,0)}{k !}(\log r+i \theta)^{k} \quad\left((r, \theta) \in E_{\rho}\right),
$$

converging on every polar-disk $E_{\rho} \equiv E((1,0), \rho)=\left\{(r, \theta) \in \mathbb{H}: \log ^{2} r+\theta^{2}<\right.$ $\left.\rho^{2}\right\}$ for all $\rho>0$. Now consider the function

$$
g(r, \theta):= \begin{cases}\frac{f(r, \theta)-f(1,0)}{\log r+i \theta} & \text { for }(r, \theta) \in \mathbb{H} \backslash\{(1,0)\}, \\ \widetilde{\Theta} f(1,0) & \text { for }(r, \theta)=(1,0) .\end{cases}
$$


In view of (12), we obviously have

$$
g(r, \theta)=\sum_{k=1}^{\infty} \frac{\widetilde{\Theta}^{k} f(1,0)}{k !}(\log r+i \theta)^{k-1}
$$

The above series has the same convergence properties as the latter. Therefore $g$ is a polar-entire function. From the definition of $g$ we see that

$$
\max _{(r, \theta) \in \partial E_{\rho}}|g(r, \theta)| \leq \frac{2 M}{\rho} \quad(\rho>0)
$$

and so, by Theorem 12, we have

$$
|g(r, \theta)| \leq \frac{2 M}{\rho} \quad \text { for }(r, \theta) \in E_{\rho} .
$$

Letting $\rho \rightarrow \infty$, we find that $g(r, \theta) \equiv 0$ on $\mathbb{H}$. This implies that $f(r, \theta)=$ $f(1,0)$ on $\mathbb{H}$, and so $f$ is constant.

As a consequence, we obtain a polar version of the fundamental theorem of algebra for "polar polynomials", using only Mellin tools achieved before.

First we note that, in general, a polynomial $P(r, \theta)$ in the variables $r$ and $\theta$ is not polar-analytic. Indeed, as an example, $P(r, \theta)=r+i \theta$ is not polar-analytic.

In view of Theorem 7, the natural analogue of polynomials in Mellin analysis are functions of the form $P(\log r+i \theta)$, where $P(z)$ is an ordinary polynomial. Indeed, we define polar polynomials of degree $n$ as functions of the form

$$
P_{n}(r, \theta):=\sum_{k=0}^{n} a_{k}(\log r+i \theta)^{k},
$$

where $a_{k}$ are complex coefficients with $a_{n} \neq 0$. We are now ready for the following result:

Theorem 14 (Fundamental Theorem of Algebra). Every polar polynomial $P_{n}$ $(r, \theta)$ of degree $n \geq 1$ has exactly $n$ zeros in $\mathbb{H}$ when counted with their multiplicities.

Proof. First assume that $P_{n}(r, \theta)$ has no zeros. Then the function

$$
f(r, \theta):=\frac{1}{P_{n}(r, \theta)}
$$

is polar-analytic on $\mathbb{H}$. Next we prove the boundedness of $f$. Clearly, $f$ is bounded on every polar disk with center at $(1,0)$. Moreover, there exists a sufficiently large $R>1$ such that if $|\log r+i \theta|>R$ then

$$
\left|P_{n}(r, \theta)\right| \geq|\log r+i \theta|^{n}\left(\left|a_{n}\right|-\frac{\left|a_{0}\right|}{|\log r+i \theta|^{n}}-\ldots-\frac{\left|a_{n-1}\right|}{|\log r+i \theta|}\right)>1 .
$$


Thus $|f(r, \theta)|<1$ outside the polar disk $E((1,0), R)$, and then $f$ is bounded on $\mathbb{H}$. Hence we can use Liouville's theorem to conclude that the function $P_{n}(r, \theta)$ is a constant, which is a contradiction since $a_{n} \neq 0$.

So far we have shown the existence of a point $\left(r_{0}, \theta_{0}\right) \in \mathbb{H}$ such that $P_{n}\left(r_{0}, \theta_{0}\right)=0$. Therefore, by Theorem 7 , we can write

$$
P_{n}(r, \theta)=\left(\log \left(r / r_{0}\right)+i\left(\theta-\theta_{0}\right)\right) Q_{n-1}(r, \theta),
$$

where $Q_{n-1}(r, \theta)$ can be shown to be a polar polynomial of degree $n-1$. Now we can apply the first part of the proof to $Q_{n-1}$, and so the assertion follows by induction.

As an interesting application of the residue theorem for polar-analytic function, we derive the following integral formula.

Theorem 15. Let $\mathscr{D} \subset \mathbb{H}$ be a domain and let $f: \mathscr{D} \rightarrow \mathbb{C}$ be polar-analytic except for isolated singularities which are all poles. Let $\gamma$ be a positively oriented, closed, regular curve that is the boundary of a simply connected domain $\operatorname{int}(\gamma) \subset \mathscr{D}$, containing a finite number of poles and zeros. Suppose that $f$ has neither a zero nor a singularity on $\gamma$. Then

$$
\frac{1}{2 \pi i} \int_{\gamma} \frac{(\widetilde{\Theta} f)(r, \theta)}{r f(r, \theta)}(d r+i r d \theta)=N-P,
$$

where $N$ is the number of zeros in $\operatorname{int}(\gamma)$ counted with their multiplicities and $P$ is the number of poles in $\operatorname{int}(\gamma)$ counted with their orders.

Proof. Let $\left(r_{0}, \theta_{0}\right)$ be a zero of $f$ of multiplicity $p$. Then we can write

$$
f(r, \theta)=\left(\log \left(r / r_{0}\right)+i\left(\theta-\theta_{0}\right)\right)^{p} g(r, \theta),
$$

with $g\left(r_{0}, \theta_{0}\right) \neq 0$. By elementary calculations, using the above representation of $f$, we deduce that

$$
\frac{\widetilde{\Theta} f(r, \theta)}{f(r, \theta)}=\frac{\widetilde{\Theta} g(r, \theta)}{g(r, \theta)}+\frac{p}{\log \left(r / r_{0}\right)+i\left(\theta-\theta_{0}\right)} .
$$

Therefore, since $g\left(r_{0}, \theta_{0}\right) \neq 0$, the function on the left-hand side has a pole of order one at $\left(r_{0}, \theta_{0}\right)$ with residue equal to $p$ in case of $c=0$.

Now let $\left(r_{0}, \theta_{0}\right)$ be a pole of order $q$. Then the function

$$
h(r, \theta):=\left(\log \left(r / r_{0}\right)+i\left(\theta-\theta_{0}\right)\right)^{q} f(r, \theta)
$$

is polar-analytic in a neighborhood of $\left(r_{0}, \theta_{0}\right)$ and $h\left(r_{0}, \theta_{0}\right) \neq 0$. Thus we can write for $(r, \theta) \neq\left(r_{0}, \theta_{0}\right)$,

$$
f(r, \theta)=\frac{h(r, \theta)}{\left(\log \left(r / r_{0}\right)+i\left(\theta-\theta_{0}\right)\right)^{q}} .
$$

Again, an elementary calculation gives

$$
\frac{\widetilde{\Theta} f(r, \theta)}{f(r, \theta)}=\frac{\widetilde{\Theta} h(r, \theta)}{h(r, \theta)}-\frac{q}{\log \left(r / r_{0}\right)+i\left(\theta-\theta_{0}\right)} .
$$


Thus the function on the left-hand side has a pole order one at $\left(r_{0}, \theta_{0}\right)$ with residue equal to $-q$ in case of $c=0$.

Now employing Theorem 11 with $c=0$ and calculating the residues with the help of (14) and (15), we obtain (13).

In particular, if no poles occur inside $\operatorname{int}(\gamma)$, we call the integral

$$
I_{\gamma}(f):=\frac{1}{2 \pi i} \int_{\gamma} \frac{\widetilde{\Theta} f(r, \theta)}{r f(r, \theta)}(d r+i r d \theta)
$$

the zeros-counting integral.

As a consequence, we establish a polar version of Rouché's theorem in its symmetric form due to Estermann (see [15, p. 156], [20, Theorem 3.6, p. 341]).

Corollary 1. Let $\mathscr{D} \subset \mathbb{H}$ be a domain and let $f, g: \mathscr{D} \rightarrow \mathbb{C}$ be polar-analytic functions. Let $\gamma$ be a positively oriented, closed, regular curve that is the boundary of a simply connected domain $\operatorname{int}(\gamma) \subset \mathscr{D}$.

If

$$
|f(r, \theta)-g(r, \theta)|<|f(r, \theta)|+|g(r, \theta)| \quad \text { for }(r, \theta) \in \gamma,
$$

then $f$ and $g$ have the same number of zeros in $\operatorname{int}(\gamma)$ counted with multiplicities.

Proof. For $t \in[0,1]$, consider the family of polar-analytic functions

$$
h_{t}(r, \theta):=t f(r, \theta)+(1-t) g(r, \theta) .
$$

Note that none of the functions $h_{t}$ vanishes on $\gamma$. Indeed, assume that $h_{0}(r, \theta)=$ 0 for some $\left(r_{0}, \theta_{0}\right) \in \gamma$. Then we have $g\left(r_{0}, \theta_{0}\right)=0$, and so (16) yields $\left|f\left(r_{0}, \theta_{0}\right)\right|<\left|f\left(r_{0}, \theta_{0}\right)\right|$; a contradiction. Analogously, $h_{1}$ cannot vanish on $\gamma$. Now, assume that there exist $\left.t^{*} \in\right] 0,1\left[\right.$ and $\left(r^{*}, \theta^{*}\right) \in \gamma$ such that $h_{t^{*}}\left(r^{*}, \theta^{*}\right)=$ 0 . Then

$$
f\left(r^{*}, \theta^{*}\right)=\frac{t^{*}-1}{t^{*}} g\left(r^{*}, \theta^{*}\right) .
$$

Substituting this in (16), we obtain

$$
\left|\frac{t^{*}-1}{t^{*}} g\left(r^{*}, \theta^{*}\right)-g\left(r^{*}, \theta^{*}\right)\right|<\frac{1-t^{*}}{t^{*}}\left|g\left(r^{*}, \theta^{*}\right)\right|+\left|g\left(r^{*}, \theta^{*}\right)\right| .
$$

The two sides are equal and so we have again a contradiction.

This shows that $\Phi(t):=I_{\gamma}\left(h_{t}\right)$ exists for all $t \in[0,1]$. It is a continuous function of $t$ and attains only integer values. Thus $\Phi$ must be a constant. Therefore, all the functions $h_{t}$ have the same number of zeros in $\operatorname{int}(\gamma)$ when counted with multiplicities. In particular this is true for $h_{0}=g$ and $h_{1}=f$.

From Corollary 1 we can deduce a polar version of the standard form of Rouché's theorem. Under the hypotheses of this corollary, suppose that

$$
|g(r, \theta)|<|f(r, \theta)| \quad \text { for }(r, \theta) \in \gamma \text {. }
$$


Setting $F:=f$ and $G:=f+g$, we easily see that the pair $(F, G)$ satisfies (16), and so $f$ and $f+g$ have the same number of zeros in $\operatorname{int}(\gamma)$ counted with multiplicities.

\section{Applications of Polar-Analytic Functions}

Here we give a survey on various interesting applications of polar analyticity in the framework of Mellin analysis.

\subsection{Function Spaces of Mellin Analysis}

Using the notion of polar-analytic function, it is possible to introduce analogues of the classical Bernstein and Hardy spaces of Fourier analysis in the setting of Mellin analysis. We begin with the definition of the Mellin-Bernstein spaces (see [3]).

For $p \in[1,+\infty], c \in \mathbb{R}$ and $T>0$, the Mellin-Bernstein space $\mathscr{B}_{c, T}^{p}$ comprises all functions $f: \mathbb{H} \rightarrow \mathbb{C}$ with the following properties:

(i) $f$ is polar-analytic on $\mathbb{H}$;

(ii) $f(\cdot, 0) \in X_{c}^{p}$;

(iii) there exists a constant $C_{f}>0$ such that $r^{c}|f(r, \theta)| \leq C_{f} e^{T|\theta|}$ for all $(r, \theta) \in \mathbb{H}$.

The spaces $\mathscr{B}_{c, T}^{p}$ characterize the spaces of functions whose Mellin transform is compactly supported on the real axis. In particular, we examine the case $p=2$. For this, we denote by $B_{c, T}^{2}$ the space of all functions $f \in X_{c}^{2} \cap C\left(\mathbb{R}^{+}\right)$ such that $[f]_{M_{c}^{2}}^{\wedge}(c+i t)=0$ for almost all $|t|>T$ and call it a Mellin-PaleyWiener space. The Paley-Wiener theorem in terms of the Mellin-Bernstein space $\mathscr{B}_{c, T}^{2}$ gives the structure of the space $B_{c, T}^{2}$ as follow (see [3, Theorem 4]).

Theorem 16 (Paley-Wiener). A function $\varphi \in X_{c}^{2}$ belongs to the Mellin-PaleyWiener space $B_{c, T}^{2}$ if and only if there exists a function $f \in \mathscr{B}_{c, T}^{2}$ such that $f(\cdot, 0)=\varphi(\cdot)$.

Another basic space is given by the so-called Mellin-Hardy space, which is an analogue of the classical Hardy spaces of Fourier analysis for functions defined on strips of the complex plane. The notion of polar-analytic function gives a simple way to define this space in Mellin setting (see [3]).

For $c \in \mathbb{R}$ and $p \in\left[1, \infty\right.$ [, we recall that the norm in $X_{c}^{p}$ is defined by

$$
\|\varphi\|_{X_{c}^{p}}=\left(\int_{0}^{+\infty}|\varphi(r)|^{p} r^{c p-1} d r\right)^{1 / p} .
$$

For a strip $\mathbb{H}_{-a, a}$ with $a>0$, we simply write $\mathbb{H}_{a}$.

Definition 4. Let $a, c, p \in \mathbb{R}$ with $a>0$ and $p \geq 1$. The Mellin-Hardy space $H_{c}^{p}\left(\mathbb{H}_{a}\right)$ comprises all functions $f: \mathbb{H}_{a} \rightarrow \mathbb{C}$ that satisfy the following conditions: 
(i) $f$ is polar-analytic on $\mathbb{H}_{a}$;

(ii) $f(\cdot, \theta) \in X_{c}^{p}$ for each $\left.\theta \in\right]-a, a[$;

(iii) there holds

$$
\|f\|_{H_{c}^{p}\left(\mathbb{H}_{a}\right)}:=\sup _{0<\theta<a}\left(\frac{\|f(\cdot, \theta)\|_{X_{c}^{p}}^{p}+\|f(\cdot,-\theta)\|_{X_{c}^{p}}^{p}}{2}\right)^{1 / p}<+\infty .
$$

When $a \in] 0, \pi]$, we can associate with each function $f \in H_{c}^{p}\left(\mathbb{H}_{a}\right)$ a function $g$ analytic on the sector $\mathscr{S}_{a}:=\{z \in \mathbb{C}:|\arg z|<a\}$ by defining $g\left(r e^{i \theta}\right):=f(r, \theta)$. The collection of all such functions constitutes a Hardytype space $H_{c}^{p}\left(\mathscr{S}_{a}\right)$, which may be identified with $H_{c}^{p}\left(\mathbb{H}_{a}\right)$.

In [14] the authors considered a Hardy space $H^{p}\left(S_{a}\right)$ of functions analytic on the strip $S_{a}:=\{z \in \mathbb{C}:|\Im z|<a\}$. The Hardy space $H_{c}^{p}\left(\mathbb{H}_{a}\right)$ has been designed in such way that if $g \in H^{p}\left(S_{a}\right)$ and

$$
f(r, \theta):=r^{-c} e^{-i c \theta} g(\log r+i \theta),
$$

then $f \in H_{c}^{p}\left(\mathbb{H}_{a}\right)$ and conversely, if $f \in H_{c}^{p}\left(\mathbb{H}_{a}\right)$ and

$$
g(x+i y):=e^{c(x+i y)} f\left(e^{x}, y\right)
$$

then $g \in H^{p}\left(S_{a}\right)$.

Theorem 16 characterizes the Mellin-Paley-Wiener spaces in terms of the Mellin-Bernstein spaces. The next theorem characterizes the space of functions whose Mellin transform has an exponential decay at infinity. In order to do that, we have to introduce a new function space that is related to Mellin-Hardy type spaces (for details see [4]).

Definition 5. Let $a, c \in \mathbb{R}$ with $a>0$ be fixed numbers. The class $H_{c}^{*}\left(\mathbb{H}_{a}\right)$ comprises all functions $f: \mathbb{H}_{a} \rightarrow \mathbb{C}$ with the following properties:

(a) $f$ is polar-analytic on $\mathbb{H}_{a}$;

(b) $\lim _{r \rightarrow 0^{+}} r^{c} f(r, 0)=\lim _{r \rightarrow+\infty} r^{c} f(r, 0)=0$;

(c) for every $\varepsilon \in] 0, a[$ there exists a constant $K(f, \varepsilon)$ such that

$$
|f(r, \theta)| \leq r^{-c} K(f, \varepsilon)
$$

for all $(r, \theta) \in \mathbb{H}_{a-\varepsilon}$;

(d) for every $\theta \in]-a, a[$ and all $t \in \mathbb{R}$,

$$
I_{c}(f, \theta, t):=\lim _{R \rightarrow+\infty} \int_{1 / R}^{R} f(r, \theta) r^{c+i t-1} d r
$$

exists and $\left|I_{c}(f, \theta, t)\right| \leq K(f)$ with a constant depending on $f$ only.

For any function $\phi: \mathbb{R}^{+} \rightarrow \mathbb{C}$ that is integrable on every compact subinterval of $\mathbb{R}^{+}$, we define

$$
M_{c}^{*}[\phi](c+i t):=\lim _{R \rightarrow+\infty} \int_{1 / R}^{R} \phi(r) r^{c+i t-1} d r .
$$


In [4], using some results concerning the $(C, 1)$-summability of the $X_{c}^{2}$-Mellin transform, it is proved that if $M_{c}^{*}[\phi]$ exists and belongs to $L^{2}(c+i \mathbb{R})$, then $\phi \in X_{c}^{2}$ and $M_{c}^{*}[\phi](c+i t)=M_{c}^{2}[\phi](c+i t)$ almost everywhere for $t \in \mathbb{R}$. This relates the functional $M_{c}^{*}$ to the $X_{c}^{2}$-Mellin transform.

Statement (d) in Definition 5 can be expressed in terms of the functional $M_{c}^{*}$ as

$$
I_{c}(f, \theta, t)=M_{c}^{*}[f(\cdot, \theta)](c+i t),
$$

and it is not difficult to show that the space $H_{c}^{*}\left(\mathbb{H}_{a}\right)$ is a normed linear space with norm

$$
\|f\|_{H_{c}^{*}\left(\mathbb{H}_{a}\right)}:=\sup \left\{\left|I_{c}(f, \theta, t)\right|: \theta \in\right]-a, a[, t \in \mathbb{R}\} .
$$

The spaces $H_{c}^{*}\left(\mathbb{H}_{a}\right)$ are related to the Mellin-Hardy spaces through the following propositions.

Proposition 4. We have

$$
H_{c}^{1}\left(\mathbb{H}_{a}\right) \varsubsetneqq H_{c}^{*}\left(\mathbb{H}_{a}\right) \quad \text { and } \quad\|f\|_{H_{c}^{*}\left(\mathbb{H}_{a}\right)} \leq 2\|f\|_{H_{c}^{1}\left(\mathbb{H}_{a}\right)} \quad \text { for } f \in H_{c}^{1}\left(\mathbb{H}_{a}\right) \text {. }
$$

Proposition 5. Let $f \in H_{c}^{*}\left(\mathbb{H}_{a}\right)$. Then, for all $\left.\alpha \in\right]-a, a[$, we have

$$
\begin{aligned}
& \quad\left|M_{c}^{*}[f(\cdot, \alpha)](c+i t)\right| \leq e^{-|t|(a-|\alpha|)}\|f\|_{H_{c}^{*}\left(\mathbb{H}_{a}\right)}, \\
& \qquad\|f(\cdot, \alpha)\|_{X_{c}^{2}} \leq \frac{1}{\sqrt{2 \pi(a-|\alpha|)}}\|f\|_{H_{c}^{*}\left(\mathbb{H}_{a}\right)} .
\end{aligned}
$$

The main theorem is as follows.

Theorem 17. A continuous function $\phi: \mathbb{R}^{+} \rightarrow \mathbb{C}$ is the restriction to $\mathbb{R}^{+}$of a function $f \in H_{c}^{*}\left(\mathbb{H}_{a}\right)$ if and only if $M_{c}^{*}[\phi]$ exists and

$$
\left|M_{c}^{*}[\phi](c+i t)\right| \leq C e^{-a|t|} \quad(t \in \mathbb{R}),
$$

with a constant $C$ that may be taken to be $\|f\|_{H_{c}^{*}\left(\mathbb{H}_{a}\right)}$.

It can be shown that the "hard part" of Theorem 16 (i.e., a function $f \in \mathscr{B}_{c, T}^{2}$ is such that $\left.f(\cdot, 0) \in B_{c, T}^{2}\right)$ can now be deduced from Theorem 17.

\subsection{Exponential Sampling}

A useful consequence of the definition of the Mellin-Bernstein spaces for polaranalytic functions is the following statement (see [3]): If $f \in \mathscr{B}_{c, T}^{p}$, then $f_{\theta}(\cdot):=$ $f(\cdot, \theta) \in X_{c}^{p}$ for every $\theta \in \mathbb{R}$. In particular, by Theorem 16 , if $f \in \mathscr{B}_{c, T}^{2}$, then $f_{\theta} \in B_{c, T}^{2}$. Thus, we can apply to $f \in \mathscr{B}_{c, T}^{2}$ the exponential sampling formula, which reconstructs the function $f$ from the sampled values of $f_{\theta}$ taken at certain sample points which are exponentially spaced over the positive real axis (see [12]). 
We premise the following definition. For $c \in \mathbb{R}$, we define the function $\operatorname{lin}_{c}: \mathbb{R}^{+} \rightarrow \mathbb{R}$ by

$$
\operatorname{lin}_{c}(x):=x^{-c} \operatorname{sinc}(\log x) .
$$

Here the sinc function is defined as $\operatorname{sinc}(x):=\frac{\sin (\pi x)}{\pi x}$ for $x \in \mathbb{R} \backslash\{0\}$ with the continuous extension $\operatorname{sinc}(0)=1$.

The exponential sampling formula for functions $f \in \mathscr{B}_{c, T}^{2}$ reads as follows:

Theorem 18. Let $f \in \mathscr{B}_{c, T}^{2}$. Then for every $\theta \in \mathbb{R}$, we have

$$
f(r, \theta)=\sum_{k \in \mathbb{Z}} f\left(e^{k \pi / T}, \theta\right) \operatorname{lin}_{c \pi / T}\left(e^{-k} r^{T}\right) .
$$

Multiplied by $r^{c}$ the series converges absolutely and uniformly on strips of bounded width parallel to the r-axis.

In particular, setting $\varphi(r):=f(r, 0)$ one has

$$
\varphi(r)=\sum_{k \in \mathbb{Z}} \varphi\left(e^{k \pi / T}\right) \operatorname{lin}_{c \pi / T}\left(e^{-k} r^{T}\right)
$$

The residue theorem (Theorem 11) is a fundamental tool for the following related result for functions $f \in \mathscr{B}_{c, T}^{p}$ with $p \in[1, \infty]$ and $c \in \mathbb{R}$ (see [7]), which is a Mellin analogue of a classical differentiation formula of Fourier analysis due to Boas (see, e.g., [14]).

Theorem 19. Let $f \in \mathscr{B}_{c, T}^{p}$, where $p \in[1,+\infty], c \in \mathbb{R}$ and $T>0$. Then

$$
\left(\widetilde{\Theta}_{c} f\right)(r, \theta)=\frac{4 T}{\pi^{2}} \sum_{k \in \mathbb{Z}} \frac{(-1)^{k}}{(2 k+1)^{2}} e^{(k+1 / 2) \pi c / T} f\left(r e^{(k+1 / 2) \pi / T}, \theta\right)
$$

for $(r, \theta) \in \mathbb{H}$. Multiplied by $r^{c}$, the series converges absolutely and uniformly on strips of bounded width parallel to the r-axis in $\mathbb{H}$.

A proof is obtained by applying the residue theorem (Theorem 11) to the function

$$
F:(r, \theta) \longmapsto \frac{f(r, \theta)}{(\log r+i \theta)^{2} \cos (\log r+i \theta)},
$$

which is polar-analytic on $\mathbb{H}$ except for isolated singularities at the points where the denominator vanishes. An important consequence of the above formula is the following Bernstein inequality for polar Mellin derivatives (see [7]).

Corollary 2. Let $f \in \mathscr{B}_{c, T}^{p}$, where $p \in[1,+\infty], c \in \mathbb{R}$ and $T>0$. Then for any $\theta \in \mathbb{R}$, we have

$$
\left\|\widetilde{\Theta}_{c} f(\cdot, \theta)\right\|_{X_{c}^{p}} \leq T\|f(\cdot, \theta)\|_{X_{c}^{p}} .
$$


In Fourier analysis, there exists a sampling formula of Valiron-sometimes also attributed to Tschakaloff (see, e.g., [17, p. 60], [14]) — that improves upon the classical sampling formula. It applies to a wider class of functions and its series converges faster. The only price one has to pay for this improvement is that, apart from the samples of the classical sampling formula, also the value of the derivative at zero is needed.

An analogue for polar-analytic functions was given in [8]. The statement is as follows.

Theorem 20. Let $f \in \mathscr{B}_{c, T}^{\infty}$, where $c \in \mathbb{R}$ and $T \in \mathbb{R}^{+}$. Then, for $r \in \mathbb{R}^{+}$,

$$
\begin{aligned}
r^{c} f(r, 0)=\sin (T \log r) & {\left[\frac{\widetilde{\Theta}_{c} f(1,0)}{T}+\frac{f(1,0)}{T \log r}\right.} \\
& \left.+T \log r \sum_{k \in \mathbb{Z} \backslash\{0\}} \frac{(-1)^{k+1} e^{k \pi c / T} f\left(e^{k \pi / T}, 0\right)}{k \pi(k \pi-T \log r)}\right] .
\end{aligned}
$$

The series converges absolutely and uniformly on compact subsets of $\mathbb{R}^{+}$.

For a proof, we may again use the residue theorem in Mellin setting, applying it to the function

$$
F:(r, \theta) \longmapsto \frac{f(r, \theta)}{(\log (r / t)+i \theta)(\log r+i \theta) \sin (\log r+i \theta)}
$$

this time.

As a final application, we now present the following exponential sampling formula that involves samples of Mellin derivatives up to the order $m$. This formula includes (21) as a special case. Since we restrict ourselves to $p=2$, we state the result for functions $f \in B_{c, T}^{2}$. The extension to $\mathscr{B}_{c, T}^{2}$ via Theorem 16 is used in the proof only. For details see [8].

Theorem 21. Let $f \in B_{c, T}^{2}$ with $c \in \mathbb{R}$ and $T>0$. Then for $m \in \mathbb{N}_{0}$ and $t \in \mathbb{R}^{+}$, there holds the representation

$$
f(t)=t^{-c} \sum_{k \in \mathbb{Z}} e^{c(m+1) k \pi / T} \sin ^{m+1}\left(\log t^{T /(m+1)}\right) \frac{\left(\Theta_{c(m+1) / T}^{m} L_{k, t}\right)\left(e^{k \pi}\right)}{m !},
$$

where

$$
L_{k, t}(r):=\frac{(-1)^{(m+1) k} f\left(r^{(m+1) / T}\right)}{\log \left(t^{T /(m+1)} r^{-1}\right) \operatorname{sinc}^{m+1}\left(\log \left(e^{-k} r^{1 / \pi}\right)\right)} .
$$

The series converges uniformly on compact subsets of $\mathbb{R}^{+}$.

For the proof in [8], the special case $T=m+1$ is considered first. Then Theorem 11 is applied to the function

$$
F_{t}(r, \theta):=\frac{\tilde{f}(r, \theta)}{(\log (r / t)+i \theta) \sin ^{m+1}(\log r+i \theta)},
$$


where $\tilde{f}(r, \theta)$ is the polar-analytic extension of $f$ to a function in $\mathscr{B}_{c, T}^{2}$ and $t$ belongs to an arbitrary but fixed compact subset of $\mathbb{R}^{+}$. The general case for arbitrary $T>0$ is deduced by an appropriate substitution.

Note that the gain of involving derivatives when $m \geq 1$ is that the required sample points have a lower density than those of formula (21).

For $m=0$ we obtain (21) while for $m=1$ we can write (24) in the following form (see [8, Corollary 6]):

$$
\begin{aligned}
f(t)=\sum_{k \in \mathbb{Z}}[ & f\left(e^{2 k \pi / T}\right) \operatorname{lin}_{c \pi / T}^{2}\left(e^{-k} t^{T /(2 \pi)}\right) \\
& +\frac{2(-1)^{k}}{T}\left(\Theta_{c} f\right)\left(\left(e^{2 k \pi / T}\right) \sin \left(\log t^{T / 2}\right) \operatorname{lin}_{2 c \pi / T}\left(e^{-k} t^{T /(2 \pi)}\right)\right]
\end{aligned}
$$

for $f \in B_{c, T}^{2}$ with $c \in \mathbb{R}, T>0$ and $t \in \mathbb{R}^{+}$.

\subsection{Quadrature Over the Positive Real Line}

The general Poisson summation formula of Mellin analysis (see [11]) can be considered as a quadrature formula for functions defined on the positive real axis just as the classical Poisson summation formula of Fourier analysis can be interpreted as a quadrature formula on the real line, see [21]. For $c \in \mathbb{R}$ and $T>0$, we denote by $B_{c, T}^{1}$ the space of all functions $f \in X_{c} \cap C\left(\mathbb{R}^{+}\right)$such that $[f]_{M_{c}}^{\wedge}(c+i t)=0$ for all $|t|>T$. For $T=2 \pi \sigma, \sigma>0$, the Mellin-Poisson summation formula gives (see [5])

$$
\int_{0}^{\infty} f(x) x^{c-1} d x=\frac{1}{\sigma} \sum_{k=-\infty}^{\infty} f\left(e^{k / \sigma}\right) e^{k c / \sigma}
$$

for every $f \in B_{c, 2 \pi \sigma}^{1}$.

When $f \notin B_{c, 2 \pi \sigma}^{1}$, formula (25) is no longer exact. However, we can write

$$
\int_{0}^{\infty} f(x) x^{c-1} d x=\frac{1}{\sigma} \sum_{k=-\infty}^{\infty} f\left(e^{k / \sigma}\right) e^{k c / \sigma}+R_{c, \sigma}[f]
$$

and say that (25) holds with a remainder term $R_{c, \sigma}[f]$. Under the validity of the general Mellin-Poisson formula, we find that

$$
R_{c, \sigma}[f]=-\sum_{k \in \mathbb{Z}, k \neq 0}[f]_{M_{c}}^{\wedge}(c+2 \pi i \sigma k) .
$$

The theory of polar-analytic functions is very useful for studying the speed of convergence to zero of the remainder and to obtain characterizations of function spaces by this speed. Results of this type were given in [5]. Here we report on results involving Mellin-Hardy spaces. Related results can be formulated for Mellin-Sobolev spaces.

We begin with an introduction of a class of functions for which the validity of the Mellin-Poisson formula is guaranteed for sufficiently large $\sigma$ with absolutely convergent series on both sides (see [11, Corollary 7.5]). Let $c \in \mathbb{R}$ 
and $s>0$. Then we denote by $\mathscr{K}_{c}(s)$ the class of all functions $f \in X_{c} \cap C\left(\mathbb{R}^{+}\right)$ for which $\Theta_{c} f$ exists almost everywhere on $\mathbb{R}^{+}$as a function in $X_{c}$ and

$$
\sum_{k=-\infty}^{\infty}\left|[f]_{M_{c}}^{\wedge}(c+i 2 \pi k \sigma)\right|<+\infty
$$

for all $\sigma \geq s$.

Now for $f \in \mathscr{K}_{c}(s)$, we denote by $f_{c+}$ and $f_{c-}$ the $c$-Mellin even and the $c$-Mellin odd parts of $f$, respectively, defined by

$$
f_{c+}(x):=\frac{1}{2}\left(x^{c} f(x)+x^{-c} f(1 / x)\right), \quad f_{c-}(x):=\frac{1}{2}\left(x^{c} f(x)-x^{-c} f(1 / x)\right) \quad(x>0)
$$

so that $f(x)=x^{-c}\left(f_{c+}(x)+f_{c-}(x)\right)$ for every $x>0$.

As was observed in [22, Proposition 4.1], for all $\sigma \geq s$, there hold the relations

$$
R_{c, \sigma}[f]=R_{0, \sigma}\left[f_{c+}\right], \quad R_{0, \sigma}\left[f_{c-}\right]=0 .
$$

Naively one might expect that $R_{c, \sigma}[f]$ converges rapidly to zero as $\sigma \rightarrow \infty$ if and only if $f$ is of high regularity. However, the previous relations show that the remainder of a $c$-Mellin odd function $f$ is zero even if its graph, apart from a symmetry, is very irregular. Therefore, in order to obtain characterizations of the speed of convergence to zero of the remainder $R_{c, \sigma}[f]$ by function spaces specified by a regularity property, we will consider the case of the Mellin-even part of functions or the case of translated functions since Mellin translations will destroy possible symmetries.

For $c \in \mathbb{R}$, we denote by $\mathscr{M}_{c}^{1}$ the space of all functions $f \in X_{c} \cap C(\mathbb{R})$ such that $[f]_{M_{c}}^{\wedge}(c+i \cdot) \in L^{1}(\mathbb{R})$. The following theorem gives a characterization of the Hardy-type spaces $H_{0}^{*}\left(\mathbb{H}_{a}\right)$ in terms of exponential (or geometric) rate of convergence (see [5, Proposition 7]).

Theorem 22. Let $\varphi \in \mathscr{K}_{c}(s) \cap \mathscr{M}_{c}^{1}$ for some $s>0$. Then for $a>0$, we have

$$
\left.\begin{array}{c}
f \in H_{0}^{*}\left(\mathbb{H}_{a}\right) \\
f(\cdot, 0) \equiv \varphi_{c+}
\end{array}\right\} \Longleftrightarrow R_{c, \sigma}[\varphi]=\mathscr{O}\left(e^{-2 \pi a \sigma}\right) \quad(\sigma \rightarrow+\infty) .
$$

This result completes and generalizes a result in [22, Theorem 6.5]. The latter theorem needed the restriction $\left.a \in] 0,2 \pi^{2}\right]$ in order to avoid the use of Riemann surfaces. With our concept of polar-analytic functions this restriction on $a$ does not arise. For translated functions we have the following result (see [5, Proposition 10]).

Theorem 23. Let $\varphi \in \mathscr{K}_{c}(s) \cap \mathscr{M}_{c}^{1}$ for some $s>0$. Then

$$
\left.\begin{array}{l}
f \in H_{c}^{*}\left(\mathbb{H}_{a}\right) \\
f(\cdot, 0) \equiv \varphi
\end{array}\right\} \Longleftrightarrow\left\{\begin{array}{l}
R_{c, \sigma}\left[\tau_{h}^{c} \varphi\right]=\mathscr{O}\left(e^{-2 \pi a \sigma}\right) \quad(\sigma \rightarrow+\infty) \\
\text { uniformly for } h \in\left[e^{-1 /(2 \sigma)}, e^{1 /(2 \sigma)}\right] .
\end{array}\right.
$$


Polynomial rates of convergence, expressed by $\mathscr{O}\left(\sigma^{-k}\right)$, can be characterized by certain Mellin-Sobolev spaces (see [5]).

Funding Open Access funding enabled and organized by Projekt DEAL.

\section{Declarations}

Conflict of interest The authors declare that they have no conflict of interest.

Open Access. This article is licensed under a Creative Commons Attribution 4.0 International License, which permits use, sharing, adaptation, distribution and reproduction in any medium or format, as long as you give appropriate credit to the original author(s) and the source, provide a link to the Creative Commons licence, and indicate if changes were made. The images or other third party material in this article are included in the article's Creative Commons licence, unless indicated otherwise in a credit line to the material. If material is not included in the article's Creative Commons licence and your intended use is not permitted by statutory regulation or exceeds the permitted use, you will need to obtain permission directly from the copyright holder. To view a copy of this licence, visit http://creativecommons. org/licenses/by/4.0/.

\section{References}

[1] Bardaro, C., Butzer, P.L., Mantellini, I.: The exponential sampling theorem of signal analysis and the reproducing kernel formula in the Mellin transform setting. Sampl. Theory Signal Image Process. 13(1), 35-66 (2014)

[2] Bardaro, C., Butzer, P.L., Mantellini, I., Schmeisser, G.: On the Paley-Wiener theorem in the Mellin transform setting. J. Approx. Theory 207, 60-75 (2016)

[3] Bardaro, C., Butzer, P.L., Mantellini, I., Schmeisser, G.: A fresh approach to the Paley-Wiener theorem for Mellin transforms and the Mellin-Hardy spaces. Math. Nachr. 290, 2759-2774 (2017)

[4] Bardaro, C., Butzer, P.L., Mantellini, I., Schmeisser, G.: A generalization of the Paley-Wiener theorem for Mellin transforms and metric characterization of function spaces. Fract. Calc. Appl. Anal. 20(5), 1216-1238 (2017)

[5] Bardaro, C., Butzer, P.L., Mantellini, I., Schmeisser, G.: Quadrature formulae for the positive real axis in the setting of Mellin analysis: sharp error estimates in terms of the Mellin distance. Calcolo 55(3), article 26, 33 p (2018)

[6] Bardaro, C., Butzer, P.L., Mantellini, I., Schmeisser, G.: Development of a new concept of polar analytic functions useful in Mellin analysis. Complex Var. Elliptic Equ. 64(12), 2040-2062 (2019)

[7] Bardaro, C., Butzer, P.L., Mantellini, I., Schmeisser, G.: Integration of polaranalytic functions and applications to Boas' differentiation formula and Bernstein's inequality in Mellin frame. Boll. Unione Mat. It. 13, 503-514 (2020)

[8] Bardaro, C., Butzer, P.L., Mantellini, I., Schmeisser, G.: Valiron's interpolation formula and a derivative sampling formula in the Mellin setting acquired via polar-analytic functions. Comput. Methods Funct. Theory. 20, 629-652 (2020) 
[9] Bertero, M., Pike, E.R.: Exponential sampling method for Laplace and other dilationally invariant transforms I. Singular-system analysis. II. Examples in photon correction spectroscopy and Frauenhofer diffraction. Inverse Probl. 7, 1-20; 21-41 (1991)

[10] Butzer, P.L., Jansche, S.: A direct approach to the Mellin transform. J. Fourier Anal. Appl. 3, 325-375 (1997)

[11] Butzer, P.L., Jansche, S.: The finite Mellin transform, Mellin-Fourier series, and the Mellin-Poisson summation formula. Rend. Circ. Mat. Palermo Serie II Suppl. 52, 55-81 (1998)

[12] Butzer, P.L., Jansche, S.: The exponential sampling theorem of signal analysis. Atti Sem. Mat. Fis. Univ. Modena Suppl. 46, 99-122 (1998), special issue dedicated to Professor Calogero Vinti

[13] Butzer, P.L., Jansche, S.: A self-contained approach to Mellin transform analysis for square integrable functions, applications. Integral Transforms Spec. Funct. 8, 175-198 (1999)

[14] Butzer, P.L., Schmeisser, G., Stens, R.L.: Basic relations valid for the Bernstein space $B_{\sigma}^{p}$ and their extensions to functions from larger spaces with error estimates in terms of their distances from $B_{\sigma}^{p}$. J. Fourier Anal. Appl. 19, 333-375 (2013)

[15] Estermann, T.: Complex Numbers and Functions. Oxford University Press, Oxford (1962)

[16] Fleming, W.: Functions of Several Variables, 2nd edn. Springer, New York (1977)

[17] Higgins, J.R.: Sampling Theory in Fourier and Signal Analysis, Foundations. Oxford University Press, Oxford (1996)

[18] Hille, E.V.: Analytic Function Theory, vol. 1. Chelsea Publ. Co., New York (1959)

[19] Kaiser, T.: The Dirichlet problem in the plane with semianalytic raw data, quasi analyticity, and o-minimal structure. Duke Math. J. 147(2), 285-314 (2009)

[20] Palka, B.P.: An Introduction to Complex Function Theory. Springer, New York (1991)

[21] Schmeisser, G.: Sampling, Gaussian quadrature, and Poisson summation formula. In: Proceedings of the 1997 International Workshop on Sampling Theory and Applications. Univ. Aveiro, Aveiro, pp. 327-332 (1997)

[22] Schmeisser, G.: Quadrature over a semi-infinite interval and Mellin transform. In: Lyubarskii, Y. (ed.) Proceedings of the 1999 International Workshop on Sampling Theory and Applications. (ISBN 82-7151-0991), Norwegian University of Science and Technology, Trondheim, pp. 203-208 (1999)

[23] Szmydt, S.: Paley-Wiener theorems for the Mellin trasformation. Ann. Polon. Math. 51(1), 313-324 (1990)

[24] Szmydt, S., Ziemian, B.: Between the Paley-Wiener theorem and the Bochner tube theorem. Ann. Polon. Math. 60(3), 299-304 (1995)

[25] Tuan, V.K.: New type Paley-Wiener theorems for the modified mutidimensional Mellin transform. J. Fourier Anal. Appl. 4, 317-328 (1998) 
Carlo Bardaro and Ilaria Mantellini

Department of Mathematics and Computer Sciences

University of Perugia

Perugia

Italy

e-mail: carlo.bardaro@unipg.it;

ilaria.mantellini@unipg.it

Paul L. Butzer

Lehrstuhl A fuer Mathematik

RWTH Aachen

Aachen

Germany

e-mail: butzer@rwth-aachen.de

Gerhard Schmeisser

Department Mathematik

FAU Erlangen-Nürnberg

Erlangen

Germany

e-mail: schmeisser@mi.uni-erlangen.de

Received: April 18, 2021.

Accepted: July 5, 2021.

Publisher's Note Springer Nature remains neutral with regard to jurisdictional claims in published maps and institutional affiliations. 